\title{
Constraints on PDFs from CDF
}

\author{
Aidan Robson * \\ Glasgow University - Department of Physics and Astronomy \\ Glasgow G12 8QQ - UK
}

Recent results from CDF that provide PDF-constraining power are presented.

\section{Introduction}

The Tevatron probes a region of $x$ and $Q^{2}$ between that accessible to HERA and that of the LHC, and thus provides important information about the content of the proton. Measurements using high- $p_{T}$ leptons from $\mathrm{W}$ and $\mathrm{Z}$ boson decays, and using jets, are sensitive to parton distribution functions (PDFs) and provide PDF-constraining power.

\section{$2 \mathrm{CDF}$}

$\mathrm{CDF}$ is a general-purpose detector with excellent tracking capability - provided by a drift chamber covering $|\eta|<1$ and extended forward by silicon detectors - and calorimetry, which extends to $|\eta|<3$. Together with muon chamber coverage for $|\eta|<1.5$, these systems allow accurate reconstruction of electrons, muons and jets.

\section{High- $p_{T}$ lepton measurements}

Events with a $\mathrm{W}$ or $\mathrm{Z}$ boson decaying to electrons or muons are distinctive and are clean to reconstruct. Inclusive $\mathrm{W}$ and $\mathrm{Z}$ cross-sections from $\mathrm{CDF}$ were early measurements that, even with a small dataset, had significant systematic uncertainties coming from PDFs [2]. With the higher statistics now collected, related measurements can provide PDF constraints.

\section{1 $\mathrm{Z}$ boson rapidity}

One such measurement is the $\mathrm{Z}$ boson rapidity, measured in the electron decay channel. The boson rapidity $y_{Z}$ is closely related to the momentum fractions $x$ of the interacting partons, and at leading order the relation $x_{1,2}=\left(m_{Z} / \sqrt{s}\right) e^{ \pm y_{Z}}$ holds exactly. By measuring the rapidity distribution the proton content is therefore probed directly.

Inclusion of the forward calorimeters allows $\mathrm{Z}$ bosons to be reconstructed over almost the full kinematic range, and it is the high-rapidity events, corresponding to one interacting parton having very low $x$ and the other high $x$, that probe the less well-known parts of the parameter space. For the highest rapidity events, where both electrons are reconstructed in the forward calorimeters, one electron candidate is required to have an associated track in order to reduce backgrounds.

Challenging aspects of the measurement are in understanding the tracking efficiencies far forward in the detector, and in the background determination.

The preliminary result is shown in Figure 1 with a NNLO calculation overlaid, normalised for shape comparison. CTEQ 6.1 PDFs are used and there is good agreement with the data.

* On behalf of the CDF Collaboration 


\subsection{Forward W bosons}

In leptonically-decaying $\mathrm{W}$ boson events the neutrino escapes the detector so, unlike in the case of the $\mathrm{Z}$ boson, the rapidity of the $\mathrm{W}$ cannot be directly reconstructed.

However different regions of rapidity may be probed using the electron decay mode by measuring the cross-section for $\mathrm{W}$ boson production separately in the central and forward calorimeters [3]. Figure 2 shows the complementary acceptances for the two sections of the calorimeter. Forward electrons are required to have associated tracks, and silicononly tracking is heavily relied upon.

The total cross-section using forward electrons is found to be consistent with the

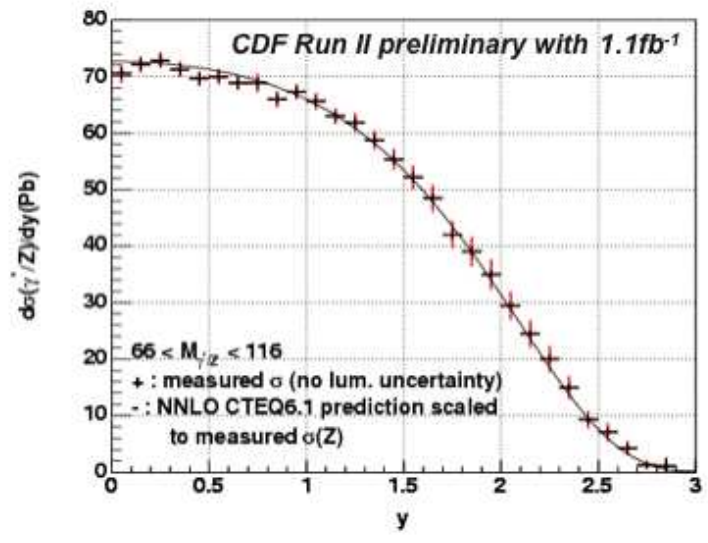

Figure 1: $\mathrm{Z}$ boson rapidity from $\mathrm{Z} \rightarrow \mathrm{ee}$ previously-measured total cross-section using central electrons. The 'visible' cross-sections are defined $\sigma_{\text {vis }}=\sigma_{\text {tot }} \times A$ where $A$ is the detector acceptance for either the central or forward regions. Then the ratio of the visible cross-sections in the two regions can be tested against theoretical predictions:

$$
\begin{aligned}
& R_{\text {exp }}=\sigma_{\text {vis }}^{\text {central }} / \sigma_{\text {vis }}^{\text {forward }}=0.925 \pm 0.033 \\
& R_{t h}=A^{\text {central }} / A^{\text {forward }}=0.924 \pm 0.030(\text { CTEQ6.1) } \\
& =0.941 \pm 0.012(\mathrm{MRST} 01 \mathrm{E})
\end{aligned}
$$

An advantage of taking the ratio is that it removes the luminosity uncertainty. The uncertainty on the measurement is comparable with the CTEQ theoretical uncertainty, and the main contribution to the measured ratio is $2.5 \%$ from the electron identification, which tends to decrease with greater statistics. This measurement is therefore promising for PDF constraining.

\subsection{W charge asymmetry}

More information about the proton content can be found by separating $\mathrm{W}$ boson events by charge. Since the proton carries on average more $u$-quark density than $d$-quark density and the antiproton more $\bar{u}$-quark density than $\bar{d}$-quark density, $\mathrm{W}^{+}$bosons tend to be produced moving in the direction of the proton beam, and $\mathrm{W}^{-}$in the antiproton direction. At a given rapidity there is therefore a non-zero $\mathrm{W}$ charge asymmetry. The asymmetry is directly related to the ratio of $d$ - to $u$-quark densities, which is otherwise not wellconstrained.

Experimentally the observable asymmetry is that of the decay leptons, which is diluted

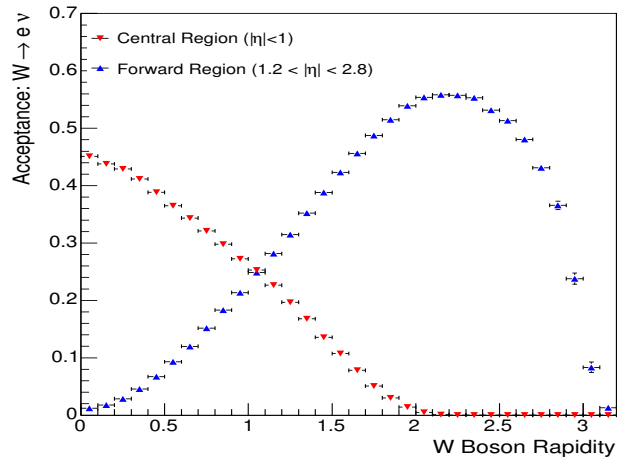

Figure 2: Central and forward $\mathrm{W}$ acceptance 
from the boson production asymmetry by the preferential emission of the charged lepton opposite to the boson direction in a V-A interaction. The first CDF Run $2 \mathrm{~W}$ charge asymmetry measurement [4] was made as a function of electron rapidity. A new approach uses all of the event kinematics to unfold directly back to the $\mathrm{W}$ boson production asymmetry [5].

By imposing a constraint on the mass of the $\mathrm{W}$ boson, two solutions can be found for the unknown longitudinal component of the neutrino momentum. Since the angular distribution of a $\mathrm{V}-\mathrm{A}$ interaction is known, the two solutions can be weighted so that the ambiguity is resolved statistically. The result is iterated to remove dependence on $d \sigma / d y$, which is an input. One of the most difficult uncertainties is the electron charge misidentification rate.

The result is given in Figure 3 with the CTEQ uncertainty band, and it is clear that the measurement should affect future fits.

\section{Jet measurements}

The inclusive jet cross-section directly probes the

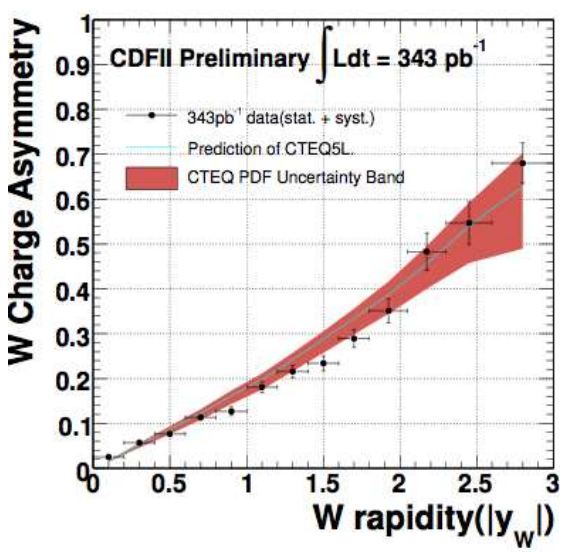

Figure 3: W charge asymmetry gluon density - the least well-known of the parton distributions. The increase of the Tevatron's centreof-mass energy from $1.8 \mathrm{TeV}$ to $1.96 \mathrm{TeV}$ between Run 1 and Run 2 has extended the reach of the inclusive jet cross-section by around $150 \mathrm{GeV}$ in $E_{T}$, and increased the cross-section by a factor of around three at $E_{T}=500 \mathrm{GeV}$.

Two jet clustering algorithms are in use: the cone-based 'midpoint' algorithm, and the $k_{T}$ algorithm, which combines proto-jets according to their separation in transverse momentum $\left(k_{T}\right)$. The midpoint algorithm is improved over a simple cone algorithm by iterating with additional seeds in between pairs of proto-jets. Overlapping jets are merged if $>75 \%$ of the energy is in the overlap region; otherwise they are split. The extra midpoint seeds provide some infra-red safety but the algorithm is still not collinear-safe. While the $k_{T}$ algorithm is more theoretically motivated, there are different systematic uncertainties associated with the two approaches and it is valuable to compare the results from each.

An important correction to the jet energy measurements is the effect of multiple protonantiproton interactions. On average there are 1.5 inelastic $\mathrm{p}-\overline{\mathrm{p}}$ interactions per bunchcrossing, but at the highest instantaneous luminosities this increases to 6 interactions per crossing. Energy is subtracted according to the number of vertices reconstructed in an event.

From Monte Carlo simulation, perturbative QCD partons and non-perturbative contributions from the underlying event and from fragmentation are reconstructed into jets. The resulting distributions can then be compared with experiment once the data has been unfolded to hadron-level distributions, using a bin-by-bin energy unfolding determined from the detector simulation.

The cross-section measurement is made in five bins of rapidity [6]. New physics is not expected to appear in the high rapidity bins so these can be used to constrain PDFs while maintaining sensitivity to new physics in the low rapidity bins. However the principal systematic uncertainty, the jet energy scale, is larger in the forward detectors. A $\pm 2-3 \%$ jet 
energy scale uncertainty translates to a $\pm 9 \%$ cross-section uncertainty at low jet $E_{T}$ and ${ }_{-40 \%}+60 \%$ at high jet $E_{T}$.

The shapes show excellent agreement over nine orders of magnitude of cross-section. The fractional uncertainties are shown for the highest rapidity bin of each analysis in Figure 4. Since experimental uncertainties are smaller than the band allowed by the current PDF fits, these measurements will improve PDF constraints, particularly on the high- $x$ gluon.
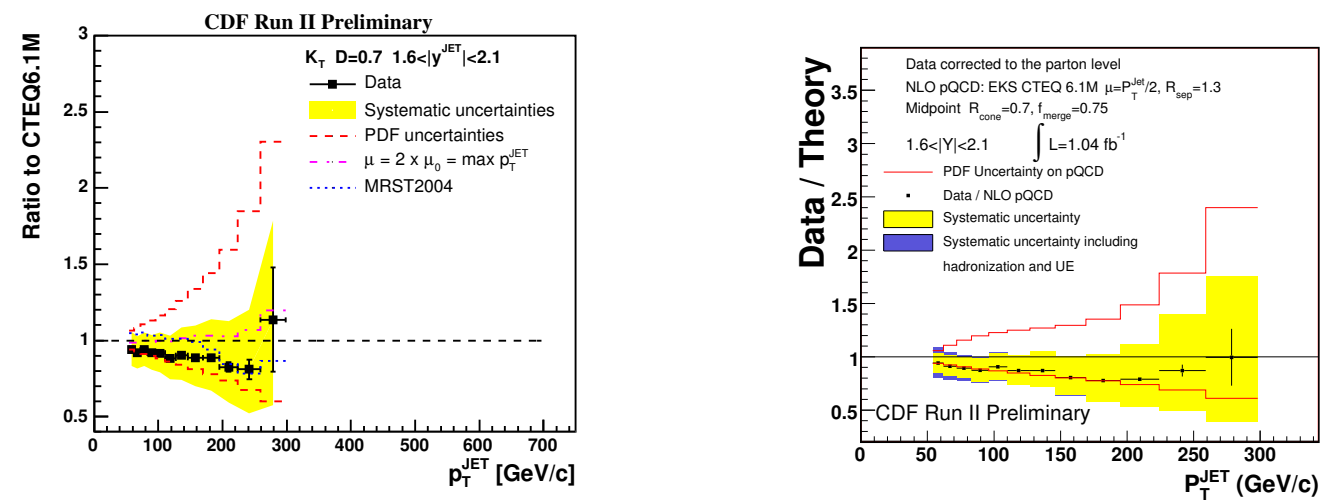

Figure 4: Inclusive jet cross-section fractional uncertainties in the highest jet rapidity bin, $1.6<|y|<2.1$ : (left) the $k_{T}$ clustering algorithm and (right) the midpoint algorithm.

\section{Conclusions}

Measurements of $\mathrm{W}$ and $\mathrm{Z}$ bosons and of the inclusive jet cross-section all provide PDF constraints that are unique to the Tevatron. All will benefit from higher statistics and will continue to be updated.

\section{Acknowledgments}

The author thanks the UK Science and Technology Facilities Council for financial support.

\section{References}

[1] Slides: http: //indico. cern. ch/contributionDisplay . py? contribId=244\&sessionId=8\& conf Id=9499

[2] D. Acosta et al., Phys. Rev. Lett. 94091803 (2005).

[3] A. Abulencia et al., arXiv:hep-ex/0702037 (2007).

[4] D. Acosta et al., Phys. Rev. D 71052002 (2005).

[5] CDF Collaboration, CDF Conference Note 8679 (2007).

[6] CDF Collaboration, CDF Conference Notes 8374, 8388 (2006). 